\title{
Charged Higgs boson production via $c b$-fusion at the Large Hadron Collider
}

\author{
J. Hernández-Sánchez $\odot^{*}$ and C. G. Honorato ${ }^{\dagger}$ \\ Fac. de Cs. de la Electrónica, Benemérita Universidad Autónoma de Puebla, \\ Apartado Postal 1152, 72570 Puebla, Puebla, México \\ S. Moretti \\ School of Physics and Astronomy, University of Southampton, \\ Highfield, Southampton SO17 1BJ, United Kingdom \\ and Particle Physics Department, Rutherford Appleton Laboratory, \\ Chilton, Didcot, Oxon OX11 OQX, United Kingdom \\ S. Rosado-Navarro ${ }^{\S}$ \\ Fac. de Cs. Físico-Matemáticas, Benemérita Universidad Autónoma de Puebla, \\ Apartado Postal 1364, C.P. 72570 Puebla, Puebla, México
}

(Received 22 April 2020; accepted 24 August 2020; published 14 September 2020)

\begin{abstract}
We analyze the production of a light charged Higgs boson at the Large Hadron Collider (LHC) via the quark-fusion mechanism $c \bar{b} \rightarrow H^{-}$considering the decay channel $H^{-} \rightarrow \tau \bar{\nu}_{\tau}$ in the final state. We study this process in the framework of the two-Higgs-doublet model type III (2HDM-III) which assumes a fourzero texture in the Yukawa matrices and a general Higgs potential, wherein the two Higgs doublets coupling to both up and down fermions do generate flavor-changing neutral currents, yet the latter can be controlled by the texture when flavor physics constraints are considered. We consider the parameter space of the model where this signal is enhanced and in agreement with both theoretical constraints and experimental data. In particular, we exploit the setup with lepton-specific-like Yukawa couplings and assess the LHC sensitivity to such $H^{ \pm}$signals against the dominant irreducible and reducible backgrounds. We show that in our model $\mathrm{BR}\left(H^{ \pm} \rightarrow c b\right) \sim 0.1-0.2$ and $\mathrm{BR}\left(H^{ \pm} \rightarrow \tau \nu\right) \sim 0.7-0.9$ so that, under these conditions the prospects for $H^{ \pm}$detection in the 2HDM-III in the aforementioned production and decay channels are excellent assuming standard collider energy and luminosity conditions.
\end{abstract}

DOI: 10.1103/PhysRevD.102.055008

\section{INTRODUCTION}

In July 2012, at the Large Hadron Collider (LHC), a neutral spinless boson was discovered by both the ATLAS [1] and CMS [2] collaborations. This new state of nature is very compatible with the Standard Model (SM) Higgs boson, so this theoretical construct now seems to be fully established. However, the SM-like limit of electroweak symmetry breaking (EWSB) dynamics induced by a Higgs potential exists in several beyond-the-SM (BSM) extensions of the Higgs sector. Notably, the two-Higgs-doublet

\footnotetext{
*jaime.hernandez@correo.buap.mx

carlosg.honorato@correo.buap.mx

s.moretti@soton.ac.uk

§sebastian.rosado@gmail.com
}

Published by the American Physical Society under the terms of the Creative Commons Attribution 4.0 International license. Further distribution of this work must maintain attribution to the author(s) and the published article's title, journal citation, and DOI. Funded by SCOAP ${ }^{3}$. model (2HDM) [3] of types I, II, III (or Y), and IV (or X), wherein flavor-changing neutral currents (FCNCs) mediated by (pseudo)scalar Higgs states can be eliminated under discrete symmetries [3], is an intriguing BSM candidate, owing to the fact that it implements the same fundamental doublet structure of the SM (in fact, twice), assumes the same SM gauge symmetry group [i.e., $\left.S U(3)_{C} \times S U(2)_{L} \times U(1)_{Y}\right]$, and predicts a variety of new Higgs boson signatures that may be accessible at the LHC. In particular, of the eight degrees of freedom pertaining to a $2 \mathrm{HDM}$, upon EWSB giving mass to the $W^{ \pm}$and $Z$ bosons, five survive as physical Higgs bosons: three are neutral (two $C P$-even, $h$ and $H$ with, conventionally, $M_{h}<M_{H}$ plus one $C P$-odd, $A$ ), while two are charged $\left(H^{ \pm}\right)$.

However, another, equally interesting kind of $2 \mathrm{HDM}$ is the one where FCNCs can be controlled by a particular texture in the Yukawa matrices [4]. In particular, in previous papers we implemented a four-zero texture in a scenario which we called 2HDM type III (2HDM-III) [5]. 
This model has a phenomenology that is very rich, which we studied at colliders in various instances [6-12], and some very interesting aspects, like flavor-violating quarks decays, which can be enhanced for neutral Higgs bosons with intermediate mass (i.e., below twice the Z-boson mass).

Furthermore, in this model the parameter space can avoid many of the current experimental constraints from flavor and Higgs physics and a light charged Higgs boson (i.e., with a mass below the top-quark mass) is allowed therein [11], so that the decay $H^{-} \rightarrow b \bar{c}$ is enhanced and its branching ratio (BR) can be dominant, above and beyond those of the customary (flavor-diagonal) $s \bar{c}$ and $\tau \nu$ channels. [In fact, this channel has also been studied in a variety of multi-Higgs doublet models (MHDMs) [13,14], wherein the $\operatorname{BR}\left(H^{-} \rightarrow b \bar{c}\right) \approx 0.7-0.8$ and one could obtain a considerable gain in sensitivity to the presence of a $H^{-}$ by tagging the $b$ quark.] Finally, we also performed a study of the process $e^{-} p \rightarrow \nu_{e} H^{-} b$ followed by the signal $H^{-} \rightarrow$ $b \bar{c}[6,15,16]$ at the Large Hadron electron Collider, finding good detection prospects.

In this work, by exploiting the enhancement of the $H^{-} \rightarrow c \bar{b}$ vertex and building on the results previously presented in Ref. [11], we study the production of a light charged Higgs boson at the LHC via heavy-quark fusion, $b \bar{c} \rightarrow H^{-}$, followed by the decay $H^{-} \rightarrow \tau \bar{\nu}_{\tau}$ (hereafter, c.c. channels are always implied). We investigate these processes in the framework of the aforementioned 2HDMIII with so-called lepton-specific couplings and assess the LHC sensitivity to this production and decay dynamics against the leading background, i.e., the irreducible one $q \bar{q}^{\prime} \rightarrow W^{-} \rightarrow \tau \bar{\nu}_{\tau}$, and the reducible noise produced by $g q^{\prime} \rightarrow W^{ \pm} q$ (with an additional jet) and $q \bar{q} \rightarrow W^{+} W^{-} \rightarrow$ $l^{+} l^{-} \nu \nu$ (where one lepton escapes detection, given that we will be looking for leptonic decays of the $\tau$ in the signal) An up-to-date overview of charged Higgs boson phenomenology at the LHC can be found in Refs. [13,17].

The plan of this paper is as follows. In the next section we describe the 2HDM-III. Then, we introduce some benchmark configurations of it for the purpose of running a Monte Carlo (MC) simulation and discussing the ensuing signal and background results. Finally, we conclude.

\section{THE 2HDM-III}

In the 2HDM-III there are two (pseudo)scalar Higgs doublets, $\Phi_{1}^{\dagger}=\left(\phi_{1}^{-}, \phi_{1}^{0 *}\right)$ and $\Phi_{2}^{\dagger}=\left(\phi_{2}^{-}, \phi_{2}^{0 *}\right)$, with hypercharge +1 , and both couple to all fermions. In order to control FCNCs, as intimated, we have implemented a specific four-zero texture as an effective flavor theory in the Yukawa sector, so that a discrete symmetry is not necessary $[10,11]$. Then, the $S U(2)_{L} \times U(1)_{Y}$-invariant scalar potential should be the most general one:

$$
\begin{aligned}
V\left(\Phi_{1}, \Phi_{2}\right)= & \mu_{1}^{2}\left(\Phi_{1}^{\dagger} \Phi_{1}\right)+\mu_{2}^{2}\left(\Phi_{2}^{\dagger} \Phi_{2}\right)-\left(\mu_{12}^{2}\left(\Phi_{1}^{\dagger} \Phi_{2}+\text { H.c. }\right)\right) \\
& +\frac{1}{2} \lambda_{1}\left(\Phi_{1}^{\dagger} \Phi_{1}\right)^{2}+\frac{1}{2} \lambda_{2}\left(\Phi_{2}^{\dagger} \Phi_{2}\right)^{2}+\lambda_{3}\left(\Phi_{1}^{\dagger} \Phi_{1}\right)\left(\Phi_{2}^{\dagger} \Phi_{2}\right)+\lambda_{4}\left(\Phi_{1}^{\dagger} \Phi_{2}\right)\left(\Phi_{2}^{\dagger} \Phi_{1}\right) \\
& +\left(\frac{1}{2} \lambda_{5}\left(\Phi_{1}^{\dagger} \Phi_{2}\right)^{2}+\lambda_{6}\left(\Phi_{1}^{\dagger} \Phi_{1}\right)\left(\Phi_{1}^{\dagger} \Phi_{2}\right)+\lambda_{7}\left(\Phi_{2}^{\dagger} \Phi_{2}\right)\left(\Phi_{1}^{\dagger} \Phi_{2}\right)+\text { H.c. }\right) .
\end{aligned}
$$

Here, we have assumed all parameters to be real, including the vacuum expectation values (VEVs) of the (pseudo) scalar fields, and therefore there is no $C P$-violating dynamics. Furthermore, note that, typically, the $\lambda_{6}$ and $\lambda_{7}$ parameters are absent when a discrete symmetry is considered (e.g., $\Phi_{1} \rightarrow \Phi_{1}$ and $\Phi_{2} \rightarrow-\Phi_{2}$ ).

Other than the physical Higgs masses $\left(M_{h}, M_{H}, M_{A}\right.$, and $M_{H}^{ \pm}$), further independent parameters of the $2 \mathrm{HDM}$ are the mixing angles $\alpha$ (related to the mass matrix of the $C P$-even sector) and $\beta$ (where $\tan \beta$ is the ratio of the two VEVs of the 2HDM). In our model, 2HDM-III, a four-zero texture is implemented as the mechanism that controls FCNCs and the terms proportional to $\lambda_{6}$ and $\lambda_{7}$ are kept. Herein, the EW parameter $\rho=M_{W}^{2} / M_{Z}^{2} \cos _{W}^{2}$ can receive corrections at the one-loop level proportional to the difference between the charged Higgs and $C P$-even/-odd masses, but it is not sensitive to the values of $\lambda_{6}$ and $\lambda_{7}$ [9]. In particular, when the difference of the scalar masses $M_{H^{ \pm}}-M_{A}\left(M_{H^{ \pm}}-M_{H}\right)$ is large, the subjacent custodial symmetry (twisted custodial symmetry) is broken. Then, a survival model to this EW observable is realised when $\rho \approx 1$ [18-20]. In general, the above mass splitting also appears in the expressions of the oblique parameters $S, T$, and $U$ [the so-called EW precision observables (EWPOs)] [21], so they should also be reconciled with the corresponding experimental bounds [22]. Hence, the benchmark scenarios chosen for our model in the next section will be in agreement with these EW measurements.

For our model the Yukawa Lagrangian is given by [11]

$$
\begin{aligned}
\mathcal{L}_{Y}= & -\left(Y_{1}^{u} \bar{Q}_{L} \tilde{\Phi}_{1} u_{R}+Y_{2}^{u} \bar{Q}_{L} \tilde{\Phi}_{2} u_{R}+Y_{1}^{d} \bar{Q}_{L} \Phi_{1} d_{R}\right. \\
& \left.+Y_{2}^{d} \bar{Q}_{L} \Phi_{2} d_{R}+Y_{1}^{l} \bar{L}_{L} \tilde{\Phi}_{1} l_{R}+Y_{2}^{l} \bar{L}_{L} \tilde{\Phi}_{2} l_{R}\right),
\end{aligned}
$$

where $\tilde{\Phi}_{1,2}=i \sigma_{2} \Phi_{1,2}^{*}$. The fermion mass matrices after EWSB are $M_{f}=\frac{1}{\sqrt{2}}\left(v_{1} Y_{1}^{f}+v_{2} Y_{2}^{f}\right), f=u, d, l$, and both 
Yukawa matrices $Y_{1}^{f}$ and $Y_{2}^{f}$ have the aforementioned fourzero texture form and are Hermitian. Once diagonalization is done, $\bar{M}_{f}=V_{f L}^{\dagger} M_{f} V_{f R}$, with $\bar{M}_{f}=\frac{1}{\sqrt{2}}\left(v_{1} \tilde{Y}_{1}^{f}+v_{2} \tilde{Y}_{2}^{f}\right)$, and $\tilde{Y}_{i}^{f}=V_{f L}^{\dagger} Y_{i}^{f} V_{f R}$, we can get from the product $V_{q} Y_{n}^{q} V_{q}^{\dagger}$ the rotated matrix $\tilde{Y}_{n}^{q}$ as [11]

$$
\left[\tilde{Y}_{n}^{q}\right]_{i j}=\frac{\sqrt{m_{i}^{q} m_{j}^{q}}}{v}\left[\tilde{\chi}_{n}^{q}\right]_{i j}=\frac{\sqrt{m_{i}^{q} m_{j}^{q}}}{v}\left[\chi_{n}^{q}\right]_{i j} e^{i \vartheta_{i j}^{q}}
$$

where the $\chi$ 's are unknown dimensionless parameters of the model. Following the procedure of Ref. [11], one can get the interactions of the charged Higgs bosons with the fermions,

$$
\begin{aligned}
\mathcal{L}^{\bar{f}_{i} f_{j} \phi}= & -\left\{\frac{\sqrt{2}}{v} \bar{u}_{i}\left(m_{d_{j}} X_{i j} P_{R}+m_{u_{i}} Y_{i j} P_{L}\right) d_{j} H^{+}\right. \\
& \left.+\frac{\sqrt{2} m_{l_{j}}}{v} Z_{i j} \bar{\nu}_{L} l_{R} H^{+}+\text {H.c. }\right\}
\end{aligned}
$$

where $X_{i j}, Y_{i j}$, and $Z_{i j}$ are defined as follows ${ }^{1}$ :

$$
\begin{gathered}
X_{i j}=\sum_{l=1}^{3}\left(V_{\mathrm{CKM}}\right)_{i l}\left[X \frac{m_{d_{l}}}{m_{d_{j}}} \delta_{l j}-\frac{f(X)}{\sqrt{2}} \sqrt{\left.\frac{m_{d_{l}}}{m_{d_{j}}} \tilde{\chi}_{l j}^{d}\right],}\right. \\
Y_{i j}=\sum_{l=1}^{3}\left[Y \delta_{i l}-\frac{f(Y)}{\sqrt{2}} \sqrt{\left.\frac{m_{u_{l}}}{m_{u_{i}}} \tilde{\chi}_{i l}^{u}\right]\left(V_{\mathrm{CKM}}\right)_{l j},}\right. \\
Z_{i j}^{l}=\left[Z \frac{m_{l_{i}}}{m_{l_{j}}} \delta_{i j}-\frac{f(Z)}{\sqrt{2}} \sqrt{\left.\frac{m_{l_{i}}}{m_{l_{j}}} \tilde{\chi}_{i j}^{l}\right],}\right.
\end{gathered}
$$

where $f(a)=\sqrt{1+a^{2}}$ and the parameters $X, Y$, and $Z$ are arbitrary complex numbers that can be linked to $\tan \beta$ or $\cot \beta$ when $\chi_{i j}^{f}=0$ [11], so that it is then possible to recover the standard four types of 2HDMs (see Table I). ${ }^{2}$ Furthermore, the Higgs-fermion-fermion couplings $(\phi f f)$ in the 2HDM-III can be written as $g_{2 \mathrm{HDM}-\mathrm{III}}^{\phi f f}=$ $g_{2 \mathrm{HDM}-\text { any }}^{\phi f f}+\Delta g$, where $g_{2 \mathrm{HDM} \text {-any }}^{\phi f f}$ is the coupling $\phi f f$ in any of the $2 \mathrm{HDMs}$ with discrete symmetry and $\Delta g$ is the contribution of the four-zero texture. Last, we also point out that this Lagrangian can represent a MHDM or an aligned 2HDM with additional flavor physics in the Yukawa matrices $[10,11]$.

\footnotetext{
${ }^{1}$ Hereafter, $V_{\mathrm{CKM}}$ is the Cabibbo-Kobayashi-Maskawa matrix.

${ }^{2}$ Hence, we will refer to these 2 HDM-III "incarnations" as 2 HDM-III like- $\chi$ scenarios, where $\chi=\mathrm{I}, \mathrm{II}, \mathrm{X}$, and Y.
}

TABLE I. The parameters $X, Y$, and $Z$ of the 2HDM-III defined in the Yukawa interactions when $\chi_{i j}^{f}=0$ so as to recover the standard four types of 2HDMs.

\begin{tabular}{lccc}
\hline \hline 2HDM-III & $X$ & $Y$ & $Z$ \\
\hline 2HDM type I & $-\cot \beta$ & $\cot \beta$ & $-\cot \beta$ \\
2HDM type II & $\tan \beta$ & $\cot \beta$ & $\tan \beta$ \\
2HDM type X & $-\cot \beta$ & $\cot \beta$ & $\tan \beta$ \\
2HDM type Y & $\tan \beta$ & $\cot \beta$ & $-\cot \beta$ \\
\hline \hline
\end{tabular}

\section{BENCHMARK SCENARIO}

We have constrained our model using flavor and Higgs physics (i.e., the measurements of the SM-like Higgs boson discovered at the LHC plus the exclusions emerging from void searches for additional Higgs states at any collider) as well as EWPOs and theoretical bounds (like vacuum stability, unitarity, and perturbativity). While we do not discuss the theoretical constraints (as they are a simple application of textbook methods), we dwell here at some length on all the experimental ones, with the intent of emphasizing those applicable to a charged Higgs state.

Specifically, the model is found to be in agreement with flavor physics constraints by taking into account the analyses performed in Refs. [10,11,23], where the parameter space of the 2HDM-III was constrained by leptonic and semileptonic meson decays, like the inclusive decays $B \rightarrow X_{s} \gamma, B_{0}-B_{0}$ as well as $K_{0}-K_{0}$ mixing and $B_{s} \rightarrow$ $\mu^{+} \mu^{-}$transitions. Here, the Yukawa texture used in the model plays a relevant role in the amplitudes of the mesonic decays, altogether allowing for the possibility to obtain a light charged Higgs state, of order $100 \mathrm{GeV}$ or so, in the case of type-X couplings (with all other Yukawa cases being more constrained in terms of $M_{H^{ \pm}}$).

Further, as for constraints from the SM-like Higgs boson measurements, we consider the impact at the one loop-level of charged Higgs bosons on the radiative decays $h \rightarrow \gamma \gamma$ and $\gamma Z$, as detailed in Ref. [9]. For this analysis, some of the most recent experimental data from the LHC are considered, namely, from Refs. [24-28]. Once again, the Yukawa texture is involved in the couplings of the charged Higgs boson with fermions in the loop and low masses for a type-X Yukawa structure are allowed.

As for the current bounds on the mass of a charged Higgs boson from direct searches at present and past colliders, we have considered the following, recalling that for a light charged Higgs boson the main production mode at lepton machines is via $e^{+} e^{-} \rightarrow H^{+} H^{-}$while at hadron colliders it is via $g g \rightarrow t \bar{b} H^{-}+$c.c. (so that, for $M_{H^{ \pm}}<m_{t}$, the latter correspond to top pair production and decay via a charged Higgs boson, i.e., $t \rightarrow b H^{+}$).

(1) LEP limits. For the mass of the charged Higgs boson, the LEP collaborations have finally established a universal lower bound at $78.6 \mathrm{GeV}$ [29]. 
(2) Tevatron limits. For a charged Higgs boson with a mass between 90 and $160 \mathrm{GeV}, \mathrm{CDF}$ and D0 established a bound for the $\mathrm{BR}\left(t \rightarrow b H^{+}\right)$of $\approx 20 \%$ taking $\mathrm{BR}\left(H^{+} \rightarrow c \bar{s}\right)=1$ or $\mathrm{BR}\left(H^{+} \rightarrow \tau^{+} \nu\right)=1$ [30-32].

(3) LHC limits. For the case $\operatorname{BR}\left(H^{+} \rightarrow \tau^{+} \nu\right)=1$ in the range of masses varying from 80 to $160 \mathrm{GeV}$, the CMS experiment has established a $\operatorname{BR}\left(t \rightarrow b H^{+}\right)=$ 2-3\% as an upper limit. Meanwhile, for the mass range 90 to $160 \mathrm{GeV}$ with $\mathrm{BR}\left(H^{+} \rightarrow c \bar{s}\right)=1$, both ATLAS and CMS set $\operatorname{BR}\left(t \rightarrow b H^{+}\right) \approx 20 \%$ as a maximum [22]. Finally, assuming $\operatorname{BR}\left(H^{+} \rightarrow c \bar{b}\right)=1$, in the mass range 90 to $150 \mathrm{GeV}$, the CMS Collaboration has set an upper limit of $\operatorname{BR}\left(t \rightarrow H^{+} b\right)=$ $0.5-0.8 \%$ [33].

As for EW data, we have fixed the oblique parameter $U=0$, because this is suppressed with respect to the parameters $S$ and $T$ when a scale for new physics (just) above the EW regime is considered [22], taking $S=0.02 \pm 0.07$ and $T=0.06 \pm 0.06$.

Upon the application of all limits above, the following parameter space region roughly survives and is analyzed here: $M_{h}=125 \mathrm{GeV}$ (thus with $h$ being the SM-like Higgs boson), $M_{A}=100 \mathrm{GeV}, \quad 180 \mathrm{GeV}<M_{H}<$ $260 \mathrm{GeV}$, and $100 \mathrm{GeV}<M_{H^{ \pm}}<170 \mathrm{GeV}$, with $0.1<$ $\cos (\beta-\alpha)<0.5$. Over such an expanse of parameter space, we consider four scenarios, each in turn being an incarnation of our 2HDM-III: like-I (where one Higgs doublet couples to all fermions); like-II (where one Higgs doublet couples to the up-type quarks and the other to the down-type quarks); like-X (also called IV or "leptonspecific," where the quark couplings are type I and the lepton ones are type II); like-Y (also called III or "flipped," where the quark couplings are type II and the lepton ones are type I).

For a light charged Higgs boson, in the 2HDM-III, the most important decay channels are $H^{ \pm} \rightarrow s c$ and $b c$, when $Y \gg X, Z$ (like-I scenario), $X, Z \gg Y$ (like-II scenario), or $X \gg Y, Z$ (like-Y scenario), in which cases the mode $H^{ \pm} \rightarrow b c$ receives a substantial enhancement coming from the four-zero texture implemented in the Yukawa matrices, so one can even get a $\operatorname{BR}\left(H^{-} \rightarrow b \bar{c}\right) \approx 0.95$. However, this does not happen for $H^{ \pm} \rightarrow \tau \nu$, which is the decay we must rely on in order to extract a charged Higgs boson signal in the hadronic environment of the LHC, specifically, assuming a leptonic decay of the $\tau$. For the case $Z \gg X, Y$ (like- $X$ scenario), the decay channel $H^{-} \rightarrow \tau \bar{\nu}_{\tau}$ is maximized, reaching a BR of $90 \%$ or so [11], while not penalizing the $H^{ \pm} \rightarrow b c$ mode excessively, so that, in turn, the production $c \bar{b} \rightarrow H^{+}$can reach a considerable cross section. In fact, a typical configuration is $\operatorname{BR}\left(H^{ \pm} \rightarrow \tau \nu\right) \approx 0.9$ and $\operatorname{BR}\left(H^{ \pm} \rightarrow c b\right) \approx 0.1$. Guided by the parameter scan performed in Ref. [8], we finally adopt the following benchmark point (BP) in order to analyze by MC simulation at the LHC the process $c \bar{b} \rightarrow H^{+} \rightarrow \tau \bar{\nu}_{\tau}$, as it offers the most optimistic chances for detection.

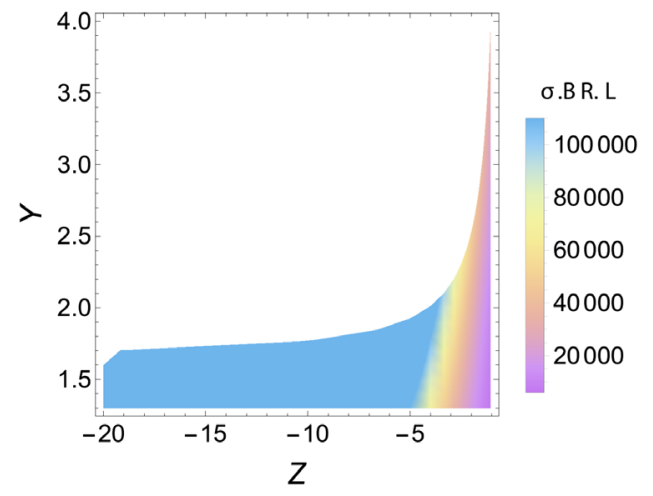

FIG. 1. Event rates for our BP at parton level for $M_{H^{ \pm}}=120 \mathrm{GeV}$ and $X=-1 / Z$, assuming $\sqrt{s}=13 \mathrm{TeV}$ and $L=36.1 \mathrm{fb}^{-1}$.

(1) Scenario 2HDM-III like-X: $\cos (\beta-\alpha)=0.5, \chi_{22}^{u}=1$, $\chi_{23}^{u}=0.1, \quad \chi_{33}^{u}=1.4, \quad \chi_{22}^{d}=1.8, \quad \chi_{23}^{d}=0.1$, $\chi_{33}^{d}=1.2, \chi_{22}^{\ell}=-0.4, \chi_{23}^{\ell}=0.1, \chi_{33}^{\ell}=1$, with $Z \gg X, Y$. Further, we assume $M_{h}=125 \mathrm{GeV}$, $M_{A}=100 \mathrm{GeV}, M_{H}=150 \mathrm{GeV}$, and $100 \mathrm{GeV}<$ $M_{H^{ \pm}}<170 \mathrm{GeV}$. In fact, eventually, given the significant signal-to-background rates obtained for a light charged Higgs boson state, we will push our analysis up to $1 \mathrm{TeV}$ or so for its mass.

\section{NUMERICAL RESULTS}

As already stressed, we will attempt to establish the signal $b \bar{c} \rightarrow H^{-} \rightarrow \tau \bar{\nu}_{\tau}$ at the LHC, by surpassing the results of Ref. [11], wherein a similar analysis was performed, although over a region of parameter space of the 2HDM-III which has largely been ruled out since, following the subsequent discovery of a SM-like Higgs boson at the LHC as well as the measurements of its properties therein. In fact, since that paper, a myriad of void experimental searches for additional Higgs bosons were also carried out by the LHC collaborations, which also impinge on the available 2HDM-III parameter space.

As intimated, the cross section for our signal process is too small in the 2HDM-III incarnations of type I, II, and Y, and therefore only the type- $\mathrm{X}$ realization is explored here. It was seen in our scan that its value is maximized for small $X$, so we fixed the latter to be $X=-1 / Z$. The results of our scan over the plane $(Y, Z)$ are presented in Fig. 1, in terms of the $\sigma\left(b \bar{c} \rightarrow H^{-}\right) \times \mathrm{BR}\left(H^{-} \rightarrow \tau \bar{\nu}_{\tau}\right) \times L$ yield, ${ }^{3}$ where $L=36.1 \mathrm{fb}^{-1}$ is the LHC luminosity at an energy of $\sqrt{s}=13 \mathrm{TeV}$, corresponding to the values used by the CMS Collaboration in their $H^{ \pm} \rightarrow \tau^{ \pm} \nu_{\tau}$ decay channel analysis [34]. Here, we fix $M_{H^{ \pm}}=120 \mathrm{GeV}$ for reference.

\footnotetext{
${ }^{3}$ In fact, here we use a factorization formula exploiting the charged Higgs boson in the narrow width approximation, given that it is very narrow. (This is done for calculation efficiency purposes.)
} 

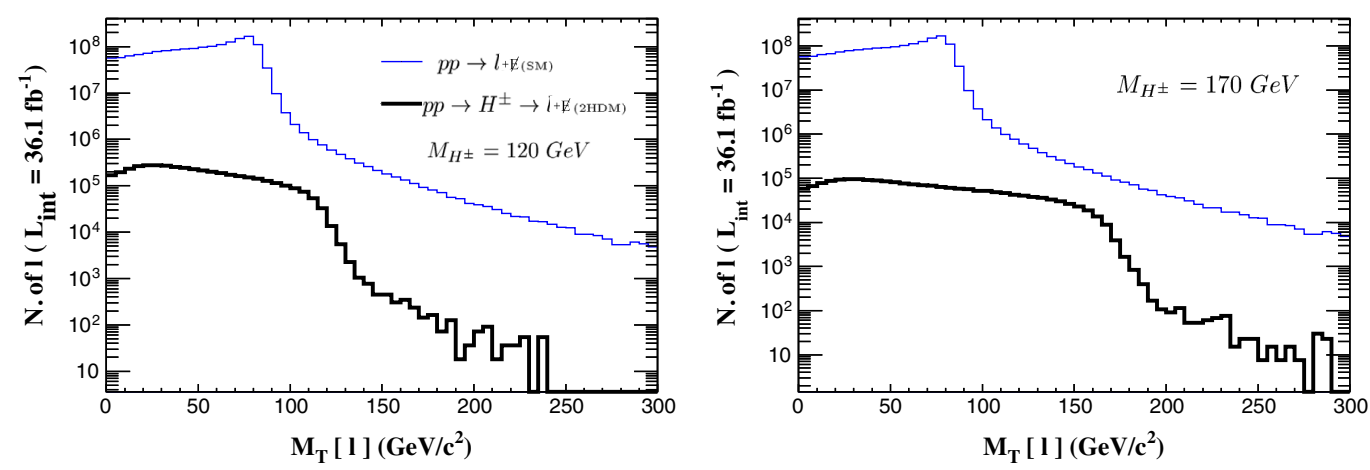

FIG. 2. Transverse mass plots for signal and background, for selected $M_{H^{ \pm}}$choices of the former. No cuts are applied here.

It is clear that the inclusive rate is very significant, with the best point being $X=0.04, Y=1.6, Z=-20$, which produces $\approx 2.276 \times 10^{6}$ events.

In order to carry out our numerical analysis, we have used CalcHEP 3.7 [35] as the parton level event generator, interfaced to the CTEQ6L1 parton distribution functions [36] and to PYTHIA6 [37] for parton shower, hadronization, and heavy flavor decays, while PGS [38] was the detector emulator, supplemented by a generic LHC parameter card. In particular, the detector parameters simulated were as follows. We considered a calorimeter coverage $|\eta|<5.0$, with segmentation $\Delta \eta \times \Delta \phi=0.087 \times 0.10$ (the number of division in $\eta$ and $\phi$ was 320 and 200, respectively). Moreover, we used a Gaussian energy resolution, with

$$
\frac{\Delta E}{E}=\frac{a}{\sqrt{E}} \oplus b,
$$

where $a=0.5$ and $b=0.03$ for both the electromagnetic and hadron calorimeter resolution, with $\oplus$ meaning addition in quadrature. The algorithm to perform jet finding was a "cone" one with jet radius $\Delta R=0.5$. The calorimeter trigger cluster finding a seed (shoulder) threshold was $5 \mathrm{GeV}$ $(1 \mathrm{GeV})$. Further, the kinematic behavior of the final-state particles was mapped with the help of MadAnalysis 5 [39].

For the MC analysis, six masses were selected for the charged Higgs boson: 120, 170, 200 400, 500, and $750 \mathrm{GeV}$. For each such value, the dominant background is the irreducible one induced by $q \bar{q}^{\prime} \rightarrow W^{ \pm} \rightarrow \tau \bar{\nu}_{\tau}$, even if $M_{H^{ \pm}}$is always significantly larger than $M_{W^{ \pm}}$(indeed, in line with the findings of Ref. [11]). The aforementioned reducible backgrounds, $g q^{\prime} \rightarrow W^{ \pm} q$ and $q \bar{q} \rightarrow W^{+} W^{-} \rightarrow$ $l^{+} l^{-} \nu \nu$, are smaller in comparison. However, all of these are included in our analysis. As previously stated, we look for leptonic $\tau$ decays, so that the final state is $l+\mathbb{E}_{T}$, where $l=e, \mu$ and $\mathscr{E}_{T}$ is the missing transverse energy. We placed no cuts on the latter, while for both lepton and jets the following acceptance region in transverse momentum and rapidity was adopted: $p_{T}(l), p_{T}(j)>10 \mathrm{GeV}$ and $|\eta(l)|,|\eta(j)|<3$ with $\Delta R(j, l)>0.5$. In fact, owing to QCD initial-state radiation, there could be any number of jets in the final state; however, in our analysis, we will finally select events with at least one lepton and no jets.

Since the invariant mass of the final state is not reconstructible (as previously shown [11]), one can analyze the transverse mass $M_{T}(l) \equiv$ $\sqrt{\left(E_{l}^{T}-\mathbb{E}_{T}\right)^{2}-\left(p_{l}^{x}+p_{\text {miss }}^{x}\right)^{2}-\left(p_{l}^{y}+p_{\text {miss }}^{y}\right)^{2}}$, where $p_{l, \text { miss }}^{x}$ and $p_{l \text {,miss }}^{y}$ are located in the transverse plane, thus assuming that the proton beams are along the $z$ axis. In Fig. 2, the shape of the transverse mass is reconstructed at detector level without selection cuts, wherein both signal and background can be seen, which reinforces the fact that, at the differential level (e.g., for $m_{H^{ \pm}}=120$ and $170 \mathrm{GeV}$, although the situation is the same for any other mass), the potential Jacobian peak correlated to the charged Higgs boson mass is well beyond the background distribution. Hence, a careful signal selection will be proposed which preserves such a difference as much as possible. In particular, we will optimize this to the given value of the charged Higgs boson mass. That is, a trial and error approach will be assumed, wherein the $M_{H^{ \pm}}$value is an input parameter to the kinematic analysis and the selection cuts adopted depend on it.

In order to fully define our selection, let us now investigate some relevant differential distributions that can be used to enhance the signal-to-background rate. (Notice that, for reasons of space, we will not show all charged Higgs mass values in each case.)

(1) From the lepton and hadronic multiplicity plots (see Figs. 3 and 4) we require at least one lepton and impose no jets in our sample. [Here, we impose on both lepton and jets the acceptance region in transverse momentum and rapidity as already discussed, i.e., $p_{T}(l), p_{T}(j)>10 \mathrm{GeV}$ and $|\eta(l)|,|\eta(j)|<3$ with $\Delta R(j, l)>0.5$.] Further, by looking at Fig. 5 (wherein the jet veto is applied), it can be seen that the cut $p_{T}(l) \geq 45 \mathrm{GeV}$ on the leptonic transverse momentum can be profitably adopted for all charged Higgs boson masses.

(2) The missing transverse energy plots (Fig. 6) suggest the use of the following cuts: for $M_{H^{ \pm}}=120 \mathrm{GeV}$, $40 \mathrm{GeV} \leq \mathscr{E}_{T} \leq 70 \mathrm{GeV}$; for $M_{H^{ \pm}}=170 \mathrm{GeV}$, 

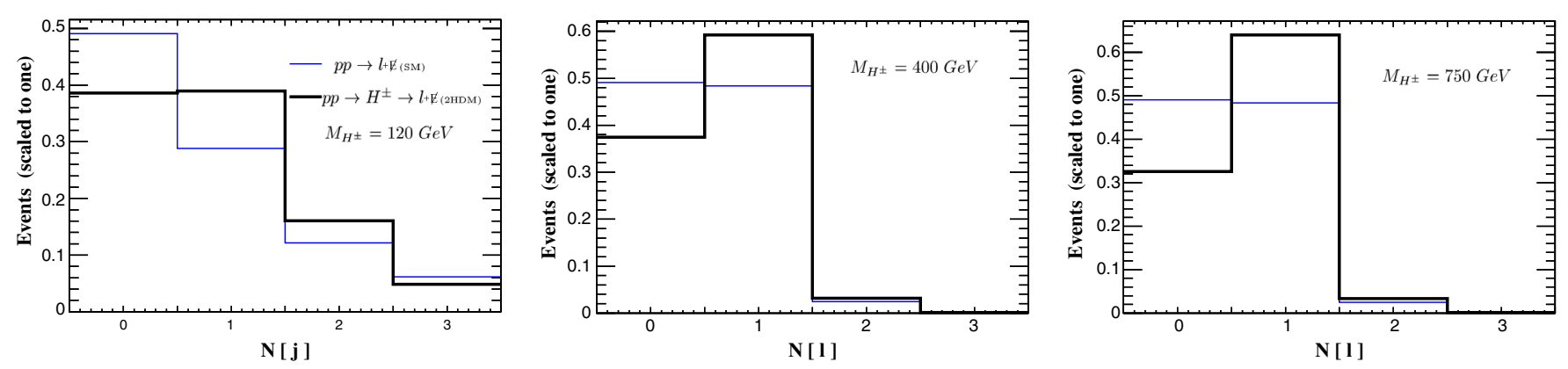

FIG. 3. Lepton multiplicity plots for signal and background, for selected $M_{H^{ \pm}}$choices of the former, over the acceptance region for leptons and jets, in both transverse momentum and pseudorapidity.
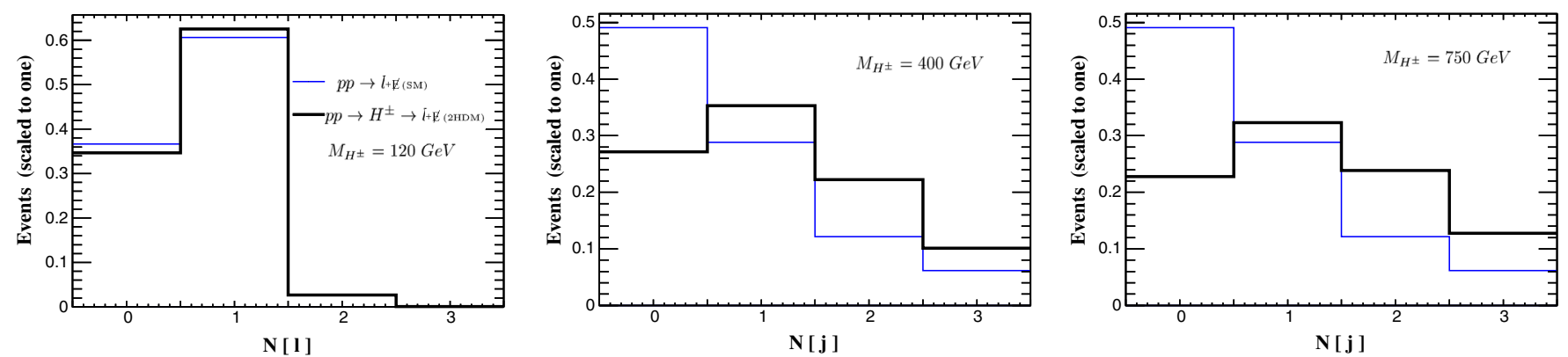

FIG. 4. Hadron multiplicity plots for signal and background, for selected $M_{H^{ \pm}}$choices of the former, over the acceptance region for leptons and jets, in both transverse momentum and pseudorapidity.
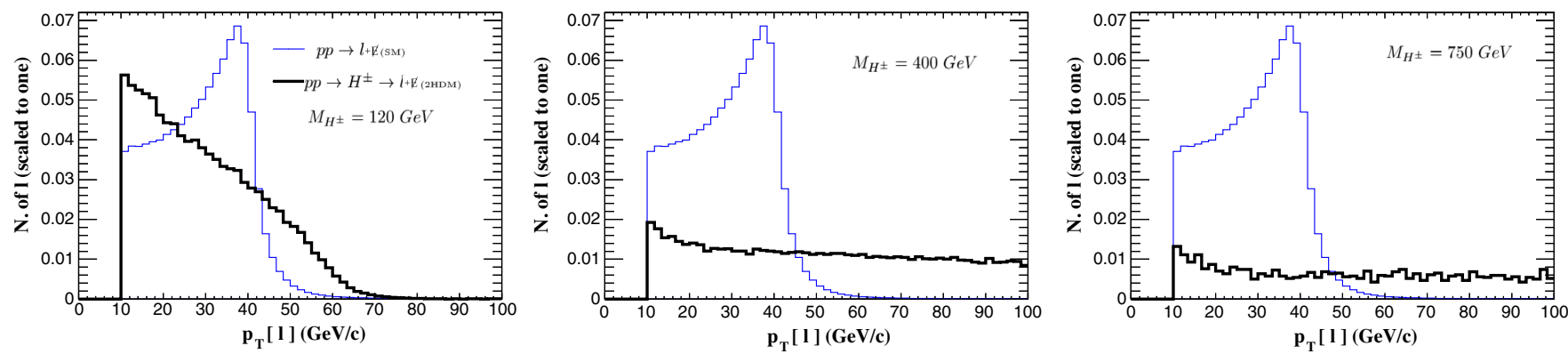

FIG. 5. Leptonic transverse momentum plots for signal and background, for selected $M_{H^{ \pm}}$choices of the former, over the acceptance region for leptons and jets, in both transverse momentum and pseudorapidity. Further, jets are vetoed here.
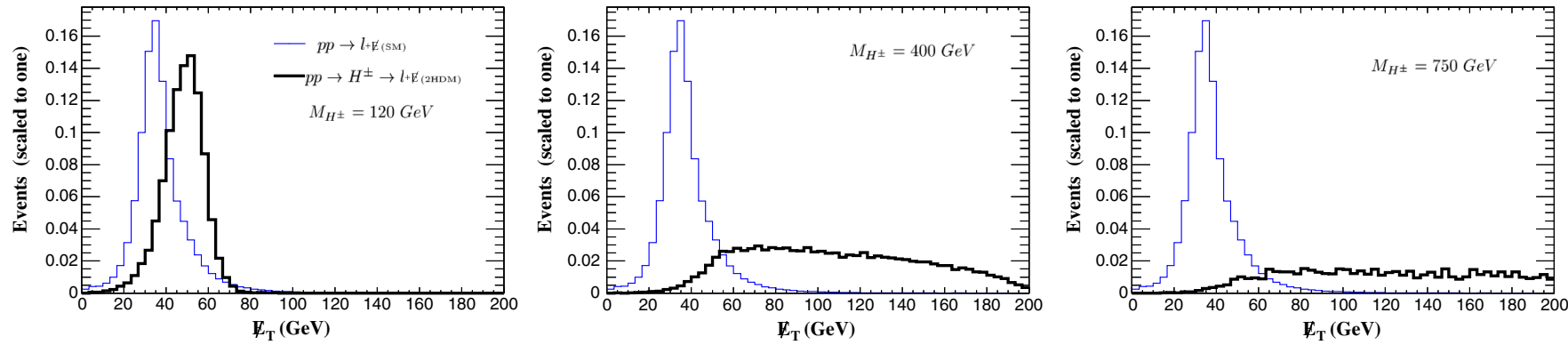

FIG. 6. Missing transverse energy plots for signal and background, for selected $M_{H^{ \pm}}$choices of the former, over the acceptance region for leptons and jets, in both transverse momentum and pseudorapidity. Further, jets are vetoed here. 
$60 \mathrm{GeV} \leq \mathscr{E}_{T} \leq 90 \mathrm{GeV} ;$ for $M_{H^{ \pm}}=200 \mathrm{GeV}$, $70 \mathrm{GeV} \leq \mathbb{E}_{T} \leq 105 \mathrm{GeV}$; for $M_{H^{ \pm}}=400 \mathrm{GeV}$, $100 \mathrm{GeV} \leq \mathbb{E}_{T} \leq 225 \mathrm{GeV}$; for $M_{H^{ \pm}}=500 \mathrm{GeV}$, $90 \mathrm{GeV} \leq \mathscr{E}_{T} \leq 270 \mathrm{GeV} ; \quad$ for $\quad M_{H^{ \pm}}=750 \mathrm{GeV}$, $105 \mathrm{GeV} \leq \mathbb{E}_{T}$.

(3) The lepton pseudorapidity (Fig. 7) shows that an optimal cut can be defined for all charged Higgs boson masses as $|\eta(l)| \leq 1.2$.

(4) The total energy (Fig. 8) shows that the following cuts can be efficient: for $M_{H^{ \pm}}=120,170 \mathrm{GeV}$, $E_{T} \geq 55 \mathrm{GeV}$; for $M_{H^{ \pm}}=200 \mathrm{GeV}, E_{T} \geq 60 \mathrm{GeV}$; for $M_{H^{ \pm}}=400 \mathrm{GeV}, E_{T} \geq 80 \mathrm{GeV}$; for $M_{H^{ \pm}}=$ $500 \mathrm{GeV}, \quad E_{T} \geq 75 \mathrm{GeV}$; for $M_{H^{ \pm}}=750 \mathrm{GeV}$, $E_{T} \geq 80 \mathrm{GeV}$.

(5) The transverse mass plots in Fig. 9 show that the last cuts to be defined are as follows: for
$M_{H^{ \pm}}=120 \mathrm{GeV}, \quad 85 \mathrm{GeV} \leq M_{T}(l) \leq 125 \mathrm{GeV} ;$ for $M_{H^{ \pm}}=170 \mathrm{GeV}, 90 \mathrm{GeV} \leq M_{T}(l) \leq 175 \mathrm{GeV}$; for $M_{H^{ \pm}}=200 \mathrm{GeV}, 110 \mathrm{GeV} \leq M_{T}(l) \leq 205 \mathrm{GeV}$; for $M_{H^{ \pm}}=400 \mathrm{GeV}, 170 \mathrm{GeV} \leq M_{T}(l) \leq 405 \mathrm{GeV}$; for $M_{H^{ \pm}}=500 \mathrm{GeV}, 200 \mathrm{GeV} \leq M_{T}(l) \leq 505 \mathrm{GeV}$; for $M_{H^{ \pm}}=750 \mathrm{GeV}, 320 \mathrm{GeV} \leq M_{T}(l) \leq 755 \mathrm{GeV}$.

Following the above sequence of cuts, for which the signal and background responses can be found in Table II, we revisit in Fig. 10 the transverse mass distributions in the relevant peak regions. From these, the significances given in Table III can be extracted. In turn, from the latter, it can be concluded that the signal is strong enough to be detectable at the LHC over a very large mass range, covering both the light- and heavy-mass regime of the charged Higgs boson stemming from the 2HDM-III. In fact, by interpolating between the various charged Higgs
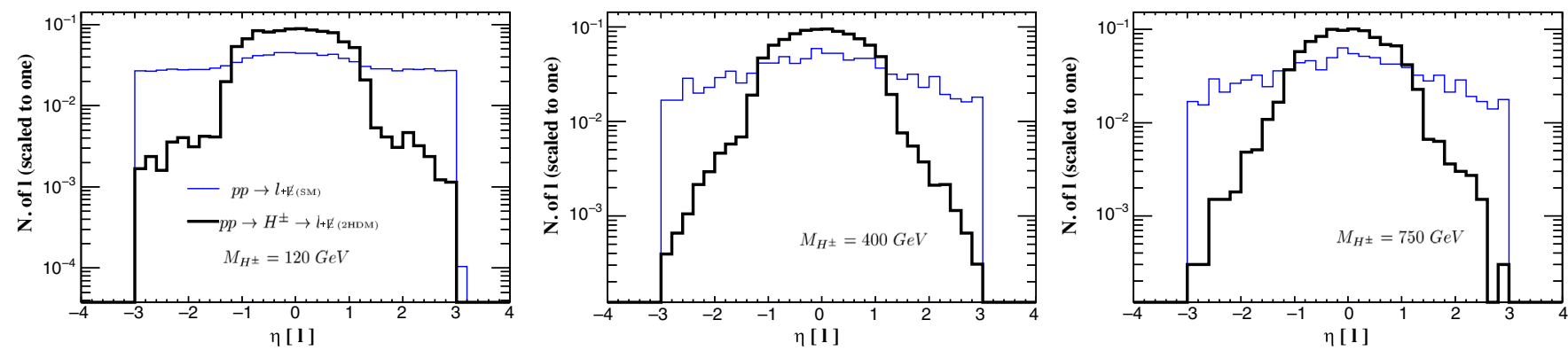

FIG. 7. Pseudorapidity plots for signal and background, for selected $M_{H^{ \pm}}$choices of the former, over the acceptance region for leptons and jets, in both transverse momentum and pseudorapidity. Further, jets are vetoed here.
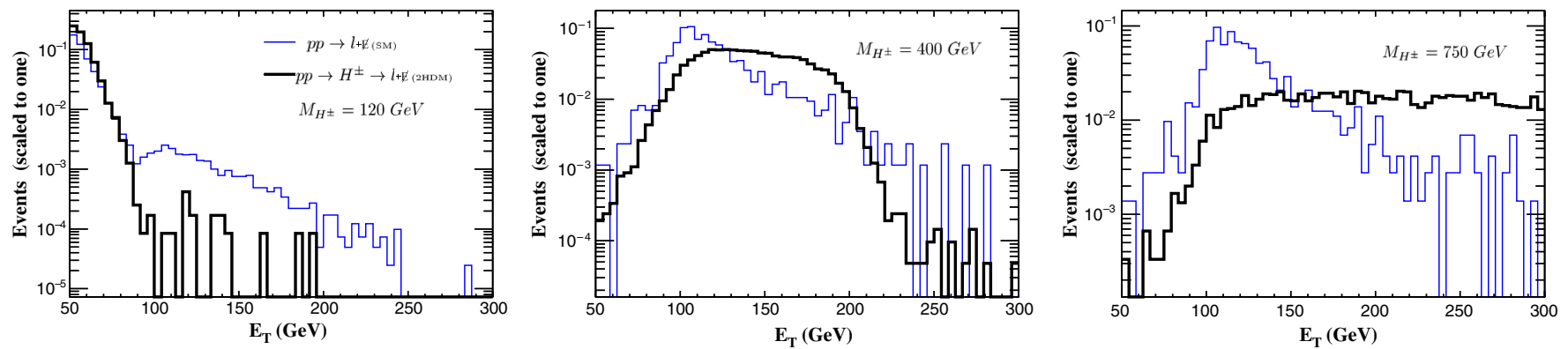

FIG. 8. Missing transverse energy plots for signal and background, for selected $M_{H^{ \pm}}$choices of the former, over the acceptance region for leptons and jets, in both transverse momentum and pseudorapidity. Further, jets are vetoed here.
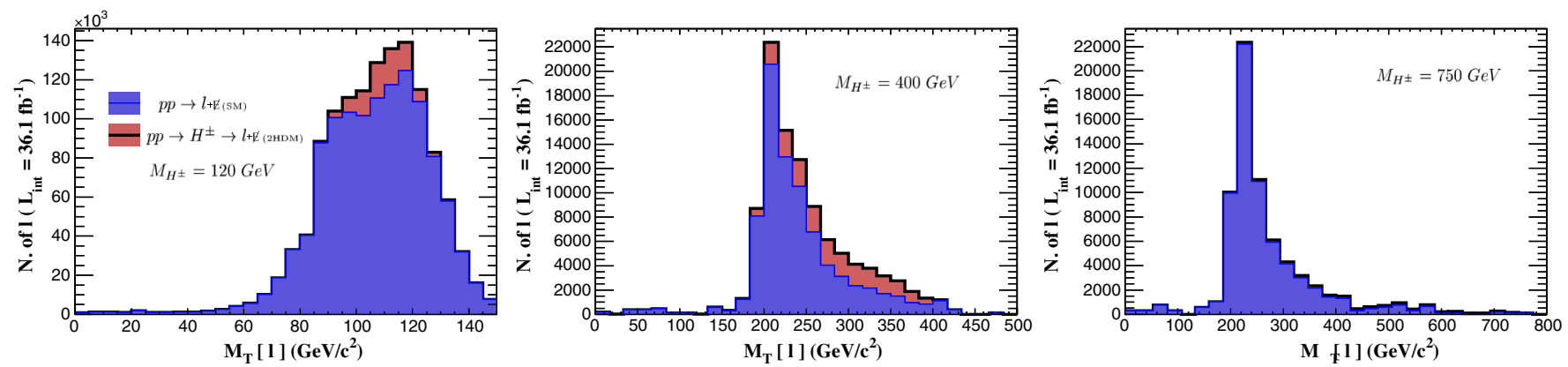

FIG. 9. Transverse mass plots for signal and background, for selected $M_{H^{ \pm}}$choices of the former, after Cuts $1-4$. Histograms are stacked here. 
TABLE II. Number of events after doing the multiplicity cuts of signal and background each cut described in the text, adopting the same sequence, $L=36.1 \mathrm{fb}^{-1}$.

\begin{tabular}{|c|c|c|c|c|c|}
\hline$M_{H^{ \pm}}$ & Cut 1: $p_{T}(l)$ & Cut 2: $\mathbb{E}_{T}$ & Cut 3: $|\eta(l)|$ & Cut 4: $E_{T}$ & Cut 5: $M_{T}(l)$ \\
\hline $120 \mathrm{GeV}$ & $\geq 45 \mathrm{GeV}$ & $\begin{array}{l}\geq 40 \mathrm{GeV} \\
\leq 70 \mathrm{GeV}\end{array}$ & $\leq 1.2 \mathrm{GeV}$ & $\geq 55 \mathrm{GeV}$ & $\begin{array}{l}\geq 85 \mathrm{GeV} \\
\leq 125 \mathrm{GeV}\end{array}$ \\
\hline Signal events & 294136 & 237167 & 215684 & 85480 & 82147 \\
\hline Background events & 27527568 & 7919086 & 3832807 & 1090294 & 795470 \\
\hline $170 \mathrm{GeV}$ & $\geq 45 \mathrm{GeV}$ & $\begin{array}{l}\geq 60 \mathrm{GeV} \\
\leq 90 \mathrm{GeV}\end{array}$ & $\leq 1.2 \mathrm{GeV}$ & $\geq 55 \mathrm{GeV}$ & $\begin{array}{l}\geq 90 \mathrm{GeV} \\
\leq 175 \mathrm{GeV}\end{array}$ \\
\hline $\begin{array}{l}\text { Signal events } \\
\text { Background events }\end{array}$ & $\begin{array}{c}290051 \\
27527568\end{array}$ & $\begin{array}{c}138676 \\
1282301\end{array}$ & $\begin{array}{l}124849 \\
669345\end{array}$ & $\begin{array}{l}114334 \\
568972\end{array}$ & $\begin{array}{l}113758 \\
536547\end{array}$ \\
\hline $200 \mathrm{GeV}$ & $\geq 45 \mathrm{GeV}$ & $\begin{array}{l}\geq 70 \mathrm{GeV} \\
\leq 105 \mathrm{GeV}\end{array}$ & $\leq 1.2 \mathrm{GeV}$ & $\geq 60 \mathrm{GeV}$ & $\begin{array}{l}\geq 110 \mathrm{GeV} \\
\leq 205 \mathrm{GeV}\end{array}$ \\
\hline $\begin{array}{l}\text { Signal events } \\
\text { Background events }\end{array}$ & $\begin{array}{c}233230 \\
27527568\end{array}$ & $\begin{array}{l}94175 \\
627241\end{array}$ & $\begin{array}{c}84981 \\
333826\end{array}$ & $\begin{array}{c}80777 \\
304128\end{array}$ & $\begin{array}{c}80453 \\
290406\end{array}$ \\
\hline $400 \mathrm{GeV}$ & $\geq 45 \mathrm{GeV}$ & $\begin{array}{l}\geq 100 \mathrm{GeV} \\
\leq 225 \mathrm{GeV}\end{array}$ & $\leq 1.2 \mathrm{GeV}$ & $\geq 80 \mathrm{GeV}$ & $\begin{array}{l}\geq 170 \mathrm{GeV} \\
\leq 405 \mathrm{GeV}\end{array}$ \\
\hline $\begin{array}{l}\text { Signal events } \\
\text { Background events }\end{array}$ & $\begin{array}{c}42612 \\
27527568\end{array}$ & $\begin{array}{c}22833 \\
146801\end{array}$ & $\begin{array}{l}20864 \\
80449\end{array}$ & $\begin{array}{l}20714 \\
78475\end{array}$ & $\begin{array}{l}20578 \\
74904\end{array}$ \\
\hline $500 \mathrm{GeV}$ & $\geq 45 \mathrm{GeV}$ & $\begin{array}{l}\geq 90 \mathrm{GeV} \\
\leq 270 \mathrm{GeV}\end{array}$ & $\leq 1.2 \mathrm{GeV}$ & $\geq 75 \mathrm{GeV}$ & $\begin{array}{l}\geq 200 \mathrm{GeV} \\
\leq 505 \mathrm{GeV}\end{array}$ \\
\hline $\begin{array}{l}\text { Signal events } \\
\text { Background events }\end{array}$ & $\begin{array}{c}20674 \\
27527568\end{array}$ & $\begin{array}{c}14716 \\
238246\end{array}$ & $\begin{array}{c}13292 \\
129132\end{array}$ & $\begin{array}{c}13194 \\
124809\end{array}$ & $\begin{array}{l}12021 \\
71238\end{array}$ \\
\hline $750 \mathrm{GeV}$ & $\geq 45 \mathrm{GeV}$ & $\geq 105 \mathrm{GeV}$ & $\geq 80 \mathrm{GeV}$ & $\geq 1 \mathrm{GeV}$ & $\begin{array}{l}\geq 320 \mathrm{GeV} \\
\leq 755 \mathrm{GeV}\end{array}$ \\
\hline $\begin{array}{l}\text { Signal events } \\
\text { Background events }\end{array}$ & $\begin{array}{c}4381 \\
27527568\end{array}$ & $\begin{array}{c}3351 \\
124057\end{array}$ & $\begin{array}{c}3049 \\
68043\end{array}$ & $\begin{array}{c}3042 \\
66539\end{array}$ & $\begin{array}{c}2279 \\
10714\end{array}$ \\
\hline
\end{tabular}
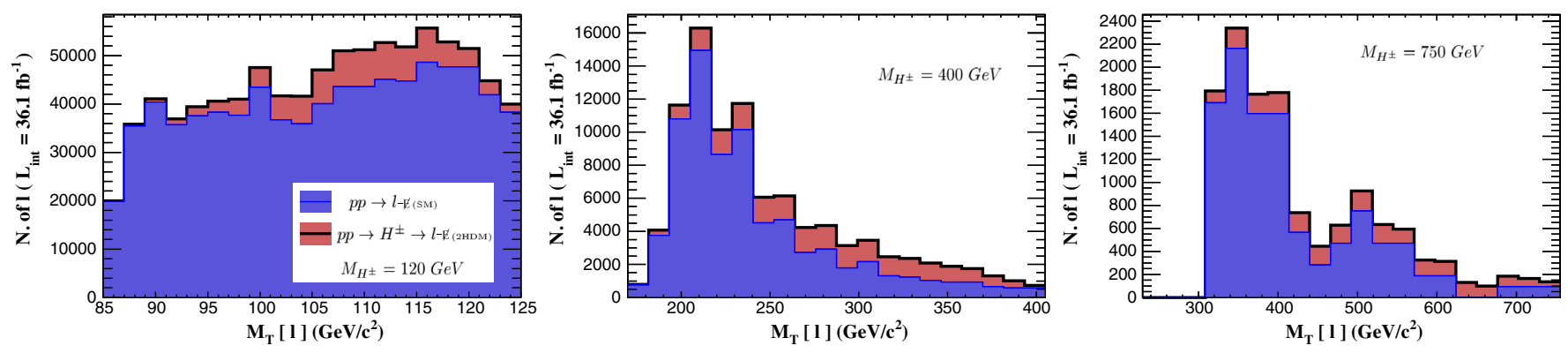

FIG. 10. Transverse mass plots for signal and background with all cuts taken into account, i.e., limited to the region used for the calculation of the significances.
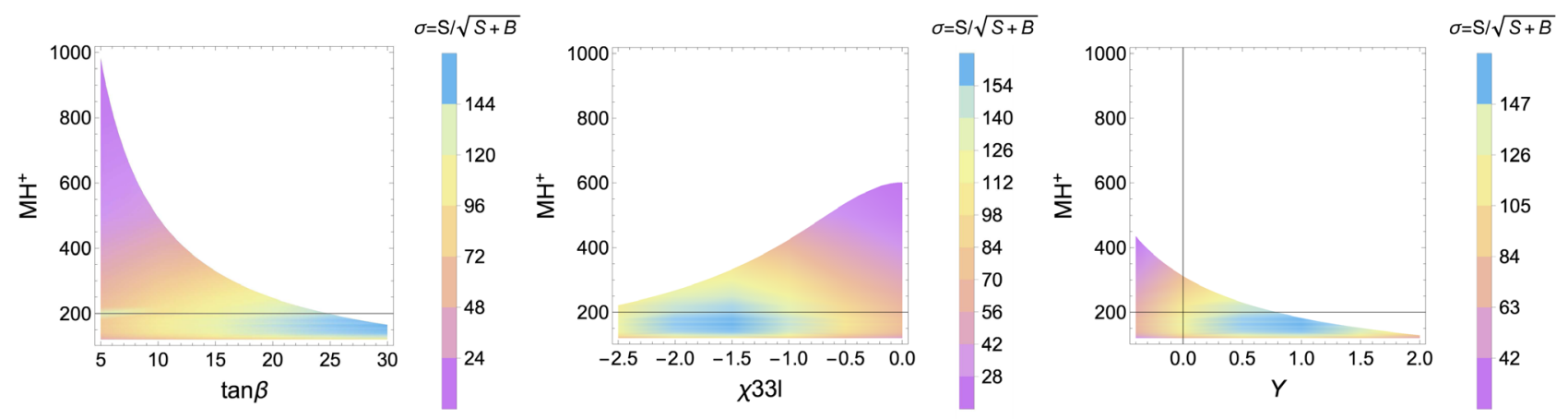

FIG. 11. Significance of our signal in terms of the most relevant parameters of the 2HDM-III like-X scenario. 
TABLE III. Significances after the complete sequence of cuts described in the text with $L=36.1 \mathrm{fb}^{-1}$.

\begin{tabular}{lrcr}
\hline \hline$H^{ \pm}$mass $(\mathrm{GeV})$ & Signal & Background & $S / \sqrt{S+B}$ \\
\hline 120 & 82147 & 795470 & 87.688 \\
130 & 111026 & 745095 & 119.994 \\
140 & 138553 & 852330 & 139.189 \\
150 & 133205 & 719156 & 144.282 \\
155 & 123148 & 633444 & 141.578 \\
160 & 131734 & 673010 & 146.849 \\
165 & 133767 & 683161 & 147.999 \\
170 & 113758 & 536547 & 141.067 \\
175 & 117716 & 544818 & 144.621 \\
180 & 121355 & 566716 & 146.299 \\
200 & 80453 & 290406 & 132.111 \\
220 & 79475 & 292568 & 130.297 \\
250 & 73119 & 314654 & 117.420 \\
300 & 38855 & 112403 & 99.906 \\
400 & 20578 & 74904 & 66.597 \\
500 & 12021 & 71238 & 41.66 \\
750 & 2279.4 & 10714 & 19.997 \\
800 & 1643.8 & 9586.2 & 15.511 \\
1000 & 637.5 & 5263 & 8.299 \\
\hline \hline
\end{tabular}

boson masses used in the MC analysis, we can perform a continuous scan of the relevant 2HDM-III like-X parameter space surviving current theoretical and experimental limits and map the signal significances, obtained at $L=$ $36.1 \mathrm{fb}^{-1}$ via the above search channel, in terms of the 2HDM-III input parameters to which the latter is sensitive, i.e., $\tan \beta, \chi_{33}^{l}$ (via $Y$ ) and $M_{H^{ \pm}}$. This is done in Fig. 11.

\section{CONCLUSIONS}

In summary, there exist significant chances to extract a charged Higgs boson signal at the LHC within the
2HDM-III scenario in its like-X incarnation by searching for the production and decay channel $b \bar{c} \rightarrow H^{-} \rightarrow \tau \bar{\nu}_{\tau}$, wherein the $\tau$ is identified through its transitions into electrons/muons and corresponding neutrinos (the latter yielding transverse missing energy). This can be achieved by the end of Run 3 over a $H^{ \pm}$mass interval ranging from $100 \mathrm{GeV}$ or so up to the TeV scale. In order to obtain this, a dedicated selection procedure is required to be optimized around a tentative charged Higgs boson mass value. We have proven this to be very effective against the (dominant) background given by $b \bar{c} \rightarrow W^{-} \rightarrow \tau \bar{\nu}_{\tau}$ as well as the (subdominant) noise produced via $g q^{\prime} \rightarrow W^{ \pm} q$ and $q \bar{q}^{\prime} \rightarrow W^{+} W^{-} \rightarrow l^{+} l^{-} \nu \nu$. Finally, we are confident that our results are realistic, as we have obtained these through a sophisticated MC analysis exploiting advanced computational tools. We are therefore looking forward to ATLAS and CMS adopting our recommended approach, so as to confirm or disprove the 2HDM-III hypothesis.

\section{ACKNOWLEDGMENTS}

S. M. is financed in part through the NExT Institute and the UK STFC Consolidated Grant No. ST/L000296/1. S. M. acknowledges support from the H2020-MSCARISE-2014 Grant No. 645722 (NonMinimalHiggs). S. R.-N. thanks the University of Southampton as well as Carleton University for hospitality while parts of this work were completed. J.H.-S. and C. H. have been supported by SNI-CONACYT (México), VIEP-BUAP and PRODEP-SEP (México) under the grant "Red Temática: Física del Higgs y del Sabor." S. R.-N. acknowledges a scholarship from CONACYT (México). We all thank Professor Heather Logan for useful and constructive discussions in the beginning of this work.
[1] G. Aad et al. (ATLAS Collaboration), Phys. Lett. B 716, 1 (2012).

[2] S. Chatchyan et al. (CMS Colllaboration), Phys. Lett. B 716, 30 (2012).

[3] G. C. Branco, P. M. Ferreira, L. Lavoura, M. N. Rebelo, M. Sher, and J. P. Silva, Phys. Rep. 516, 1 (2012).

[4] H. Fritzsch and Z.Z. Xing, Phys. Lett. B 555, 63 (2003).

[5] J. L. Díaz-Cruz, J. Hernández-Sánchez, S. Moretti, R. Noriega-Papaqui, and A. Rosado, Phys. Rev. D 79, 095025 (2009).

[6] J. Hernández-Sánchez, O. Flores-Sánchez, C. G. Honorato, S. Moretti, and S. Rosado, Proc. Sci., CHARGED2016 (2017) 032 [arXiv:1612.06316].
[7] J. Hernández-Sánchez, S. P. Das, S. Moretti, A. Rosado, and R. Xoxocotzi-Aguilar, Proc. Sci., DIS2015 (2015) 227 [arXiv:1509.05491].

[8] S. P. Das, J. Hernández-Sánchez, S. Moretti, A. Rosado, and R. Xoxocotzi, Phys. Rev. D 94, 055003 (2016).

[9] A. Cordero-Cid, J. Hernández-Sánchez, C. G. Honorato, S. Moretti, M. A. Pérez, and A. Rosado, J. High Energy Phys. 07 (2014) 057.

[10] O. Félix-Beltrán, F. González-Canales, J. HernándezSánchez, S. Moretti, R. Noriega-Papaqui, and A. Rosado, Phys. Lett. B 742, 347 (2015).

[11] J. Hernández-Sánchez, S. Moretti, R. Noriega-Papaqui, and A. Rosado, J. High Energy Phys. 07 (2013) 044. 
[12] J. Hernández-Sánchez, S. Moretti, R. Noriega-Papaqui, and A. Rosado, Proc. Sci., CHARGED2012 (2012) 029 [arXiv: 1302.0083].

[13] A. G. Akeroyd et al., Eur. Phys. J. C 77, 276 (2017).

[14] A. G. Akeroyd, S. Moretti, and J. Hernández-Sánchez, Phys. Rev. D 85, 115002 (2012).

[15] O. Flores-Sánchez, J. Hernández-Sánchez, C. G. Honorato, S. Moretti, and S. Rosado-Navarro, Phys. Rev. D 99, 095009 (2019).

[16] O. Flores-Sánchez, J. Hernández-Sánchez, C. G. Honorato, S. Moretti, and S. Rosado, Proc. Sci., DIS2019 (2019) 094 [arXiv:1908.09405].

[17] A. Arhrib, R. Benbrik, H. Harouiz, S. Moretti, and A. Rouchad, Front. Phys. 8, 39 (2020).

[18] J. F. Gunion and H.E. Haber, Phys. Rev. D 67, 075019 (2003).

[19] J.-M. Gerard and M. Herquet, Phys. Rev. Lett. 98, 251802 (2007).

[20] S. de Visscher, J. M. Gerard, M. Herquet, V. Lemaitre, and F. Maltoni, J. High Energy Phys. 08 (2009) 042.

[21] S. Kanemura, Y. Okada, H. Taniguchi, and K. Tsumura, Phys. Lett. B 704, 303 (2011).

[22] M. Tanabashi et al. (Particle Data Group), Phys. Rev. D 98, 030001 (2018).

[23] A. Crivellin, C. Greub, and A. Kokulu, Phys. Rev. D 87, 094031 (2013).

[24] M. Aaboud et al. (ATLAS Collaboration), Phys. Lett. B 784, 345 (2018).

[25] M. Aaboud et al. (ATLAS Collaboration), Phys. Lett. B 786, 114 (2018).
[26] M. Aaboud et al. (ATLAS Collaboration), J. High Energy Phys. 10 (2017) 112.

[27] A. M. Sirunyan et al. (CMS Collaboration), Eur. Phys. J. C 79, 421 (2019).

[28] A. M. Sirunyan et al. (CMS Collaboration), J. High Energy Phys. 11 (2018) 152.

[29] S. Schael et al. (ALEPH and DELPHI and L3 and OPAL Collaborations and LEP Working Group for Higgs Boson Searches), Eur. Phys. J. C 73, 2463 (2013).

[30] B. Abbott et al. (D0 Collaboration), Phys. Rev. Lett. 82, 4975 (1999).

[31] A. Abulencia et al. (CDF Collaboration), Phys. Rev. Lett. 96, 042003 (2006).

[32] V. M. Abazov et al. (D0 Collaboration), Phys. Lett. B 682, 278 (2009).

[33] A. M. Sirunyan et al. (CMS Collaboration), J. High Energy Phys. 11 (2018) 115.

[34] A. M. Sirunyan et al. (CMS Collaboration), J. High Energy Phys. 07 (2019) 142.

[35] A. Belyaev, N. D. Christensen, and A. Pukhov, Comput. Phys. Commun. 184, 1729 (2013).

[36] J. Pumplin, D. R. Stump, J. Huston, H. L. Lai, P. M. Nadolsky, and W. K. Tung, J. High Energy Phys. 07 (2002) 012.

[37] T. Sjöstrand, S. Mrenna, and P. Z. Skands, J. High Energy Phys. 05 (2006) 026.

[38] J. Conway, R. Culbertson, R. Demina, B. Kilminster, M. Kruse, S. Mrenna, J. Nielsen, M. Roco, A. Pierce, J. Thaler, and T. Wizansky, http://conway.physics.ucdavis.edu/ research/software/pgs/pgs4-general.htm.

[39] E. Conte, B. Fuks, and G. Serret, Comput. Phys. Commun. 184, 222 (2013). 